\title{
Muscle twitch activity during REM sleep: Effect of sleep deprivation and relation with rapid eye movement activity
}

\author{
LUIGI DE GENNARO, MICHELE FERRARA, and MARIO BERTINI \\ University of Rome "La Sapienza," Rome, Italy
}

\begin{abstract}
The present study assessed the effect of total sleep deprivation on muscle twitch (MT) frequency during the recovery night, as well as the intranight distribution of MTs and their correlation with rapid eye movements (REMs) in the baseline nights of a larger sample of subjects. Results showed that, in the recovery night, sleep deprivation determined an increase of slow-wave sleep amount and of sleep efficiency and a decrease of Stage 1, Stage 2, and intrasleep wake. In the same night, MT and REM frequency decreased, as compared with the baseline night. As regards intranight distribution, MT frequency showed a slight increase in the third and fourth cycles, as compared with the first one, whereas REM frequency sharply increased during the second, third, and fourth REM cycles, as compared with the first one. Finally, a weak but significant correlation between REMs and MTs was found only in the first REM cycle.
\end{abstract}

Since the discovery of REM sleep, several studies have focused on the most characteristic phasic feature of REM sleep, the rapid eye movements (REMs). Aserinsky (1973) was the first to hypothesize that REMs might be an index of sleep need or "satiety" and suggested the existence of an inverse relationship between oculomotor activity during REM sleep and sleep depth. This hypothesis has been supported by the decrease of REMs found after total sleep deprivation (Feinberg, Floyd, \& March, 1987; Reynolds et al., 1986). An inverse relationship between REMs and sleep depth and/or sleep need has been confirmed also by experiments showing a significant reduction of REM frequency after a night in which sleep was restricted to 100 min (Feinberg, Baker, Leder, \& March, 1988) or cut short by $4 \mathrm{~h}$ (Travis, Maloney, Means, March, \& Feinberg, 1991). More recently, a within-subjects study showed, in the recovery night following a gradual sleep restriction, a decreased REM frequency linearly related to the amount of sleep curtailment and to slow-wave sleep (SWS) rebound (Lucidi et al., 1996). Finally, a decrease of REM frequency has been found in recovery sleep after selective SWS deprivation without any sleep curtailment; in this case, REM frequency was independent of prior sleep duration and also negatively correlated with SWS amount more than with arousal thresholds (De Gennaro, Ferrara, \& Bertini, 2000).

Other minor muscle movements occur during REM sleep within the general muscle atonia that tonically char-

Thanks to Simona Baldanza for her invaluable help in data collecting. Correspondence concerning this article should be addressed to L. De Gennaro, Dipartimento di Psicologia, Sezione di Neuroscienze, Università degli Studi di Roma "La Sapienza," Via dei Marsi, 78, 00185 Roma, Italy (e-mail: luigi.degennaro@uniromal.it). acterizes this sleep stage, including middle-ear muscle activity (MEMA) and muscle twitches (MTs). It has been recently found that MEMA does not parallel the decrease of REM frequency after a night of total sleep deprivation (De Gennaro \& Ferrara, 2000), whereas, to the best of our knowledge, no data have ever been provided on MT activity after total sleep deprivation. Furthermore, little is known about two other issues concerning MTs during REM sleep: (1) a possible association between MTs and REMs and (2) the existence of a progressive increase in MT activity across subsequent sleep cycles. Only two studies have addressed these issues, providing not entirely consistent results. No correlation has been found between MTs and REMs in small samples of narcoleptics (Geisler, Meier-Ewert, \& Matsubayshi, 1987; Tafti, Olivet, \& Billiard, 1991), apneics (Tafti et al., 1991), and normal subjects (Geisler et al., 1987). As regards intranight distribution of MTs, Geisler and co-workers reported a significant increase of relative MT density from the first to the second REM period in normal subjects, with no further change during the rest of the night, and a decrease from the first to the second REM period in narcoleptics. On the other hand, Tafti and co-workers reported no significant difference in relative MT density across the night, either in narcoleptics or in normal controls, and a significant decrease of relative MT density from the first REM period to the second and third ones in apneics.

The aims of the present study are (1) to assess MT activity after $40 \mathrm{~h}$ of total sleep deprivation (as a consequence of the homeostatic increase of SWS, we hypothesize a decrease of MTs during recovery sleep, as compared with baseline, paralleling the already reported REM frequency decrease; De Gennaro \& Ferrara, 2000) and (2) to further evaluate the intranight variations of MTs and their correlation with REMs in a larger sample of subjects. 
Table 1

Means, Standard Deviations, and ANOVA Results of the Sleep Variables During Baseline (BSL) and Recovery (REC) Nights

\begin{tabular}{|c|c|c|c|c|c|c|}
\hline \multirow[b]{2}{*}{ Sleep Variables } & \multicolumn{2}{|c|}{ BSL } & \multicolumn{2}{|c|}{ REC } & \multicolumn{2}{|c|}{ ANOVA } \\
\hline & $\underline{M}$ & $S D$ & $M$ & $S D$ & $F(1,8)$ & $p$ \\
\hline Stage 1 duration & 36.7 & 15.4 & 19.8 & 8.3 & 15.42 & .004 \\
\hline Stage 2 duration & 256.0 & 25.8 & 228.8 & 39.9 & 7.53 & .02 \\
\hline SWS duration & 49.2 & 25.7 & 99.0 & 42.4 & 23.70 & .001 \\
\hline REM duration & 107.7 & 24.8 & 99.9 & 8.6 & 0.82 & .39 \\
\hline ISW duration & 17.3 & 7.5 & 9.3 & 4.3 & 5.88 & .04 \\
\hline SE index & 92.5 & 4.6 & 97.2 & 0.9 & 9.33 & .02 \\
\hline TST & 449.6 & 21.2 & 447.6 & 17.8 & 0.21 & .65 \\
\hline
\end{tabular}

Note-All durations are expressed in minutes. SWS, slow-wave sleep (Sleep Stages $3+$ 4); ISW, intrasleep wake; SE, sleep efficiency index (total bed time/total sleep time $\times$ 100 ); TST, total sleep time (in minutes).

\section{METHOD}

\section{Subjects}

Nine normal male subjects (mean age, 23.2 years) were selected as paid volunteers for the study involving total sleep deprivation. The requirements for inclusion were normal sleep duration and schedule, no daytime nap habits, no excessive daytime sleepiness, and no other sleep, medical, or psychiatric disorder, as assessed by a 1 -week sleep log and by a clinical interview. Each subject was unaware of the purpose of the experiment and gave informed consent.

\section{Procedure}

The subjects slept in a soundproof, temperature-controlled room for three nights: (1) adaptation, (2) baseline, and (3) recovery. Every night, sleep recording started at about 11:30 p.m. and ended after $7.5 \mathrm{~h}$ of accumulated sleep. In the adaptation and baseline nights, each subject arrived at the sleep laboratory at 9:00 p.m., and after electrode montage, his undisturbed sleep was recorded. A 40-h schedule of sleep deprivation began after awakening on the morning following the baseline night. The subjects remained in the sleep laboratory together with at least one experimenter throughout the $40 \mathrm{~h}$ of continuous wakefulness. Every $2 \mathrm{~h}$, variations of alertness and performance were assessed by means of subjective ratings of sleepiness, behavioral measures, and oculomotor activity (smooth pursuit, saccadic eye movements, and optokinetic responses). Each testing session lasted $30 \mathrm{~min}$. Data on these measures will be reported elsewhere.

The subject's meals were scheduled at $8: 30,14: 30$, and 19:30; nonscheduled light snacks were permitted. The 40 -h schedule of sleep deprivation ended at 10:30 p.m.; the recording of the recovery night began at about 11:30 p.m. The subjects were awakened after $7.5 \mathrm{~h}$ of accumulated sleep.

\section{Sleep Recording}

A VEGA 24 (Esaote Biomedica, Firenze) polygraph set at a paper speed of $10 \mathrm{~mm} / \mathrm{sec}$ was used for polygraphic recordings. Electroencephalograms (EEGs; C3-A2 and C4-A1) were recorded with an $\mathrm{AC}$ time constant of $0.3 \mathrm{sec}$. Bipolar horizontal and vertical eye movements were recorded with a time constant of $1 \mathrm{sec}$. Bipolar horizontal electrooculograms (EOGs) were recorded from electrodes placed about $1 \mathrm{~cm}$ from the medial and lateral canthi of the dominant eye, and bipolar vertical EOGs from electrodes located about $3 \mathrm{~cm}$ above and below the right eye pupil.

Two submental electromyographs (EMGs) were recorded with a time constant of $0.03 \mathrm{sec}$ as a standard measure of EMG activity during sleep; supplemental bipolar EMGs, with a time constant of $0.03 \mathrm{sec}$, were recorded by right and left masseter muscle elec- trodes. One submental EMG electrode was affixed beneath the chin, following the standard procedure for sleep research (Rechtschaffen \& Kales, 1968); electrodes recording the other submental EMGs were placed $1 \mathrm{~cm}$ posterior to the standard submental electrode. Right and left masseter EMG electrodes were affixed in accordance with the guidelines for human EMG research (Fridlund \& Cacioppo, 1986): The first electrode was situated along an imaginary line extending from the gonion (the most lateral point on the mandibular angle) to the exocanthion (the lateral commissure of the eye fissure), $2 \mathrm{~cm}$ from the gonion; the second electrode was placed $1 \mathrm{~cm}$ superior and slightly lateral to the first. Electrode impedance was kept below $5 \mathrm{k} \Omega$.

\section{Data Analysis}

Left central EEGs (C3-A2), EMGs, and horizontal and vertical EOGs were used to visually score sleep stages, according to the standard criteria (Rechtschaffen \& Kales, 1968). With regard to SWS scoring, the amplitude criterion $(>75 \mu \mathrm{V})$ was strictly followed.

The EOG was scored visually by two experimenters, who were blind with respect to the experimental condition. Cases in which the two scorers were in disagreement were resolved by a third blind scorer. Any deflection of the pen equivalent to an REM greater than $3^{\circ}$ and separated from another deflection by more than $200 \mathrm{msec}$ was considered to be an independent eye movement. The minimal velocity for considering an eye movement as "rapid" was $50 \mathrm{deg} / \mathrm{sec}$ (Ktonas, Wu, \& Renault, 1992). REM frequency was expressed by the ratio number of REMs/duration of each REM period (in minutes).

MT activity was also scored visually by two experimenters, blind with respect to the experimental condition; cases in which they were in disagreement were resolved by a third blind scorer. MT activity was scored in accordance with the criteria proposed by Geisler et al. (1987). Any EMG deflection was scored as an MT when (1) its duration was shorter than $1 \mathrm{sec},(2)$ its amplitude was at least $20 \mu \mathrm{V}$ and exceeded three times the average EMG amplitude within an interval of $1 \mathrm{~min}$, (3) there was no change in muscle tone or movement arousals before and after a 3-sec interval, and (4) there was no other artifact. In addition, an interval greater than $500 \mathrm{msec}$ was required between any two EMG deflections for them to be considered as independent MTs.

MT frequency was expressed by the ratio number of valid MT deflections/duration of each REM period (in minutes). To normalize their distribution and to reduce proportionality of means and variances, MT frequency values were $\log$ transformed $[\log (X+1)]$ before statistical analysis.

To assess the time course of MTs and REMs across REM periods, as well as the intercorrelations between these two measures, the baseline nights of the above-mentioned 9 subjects and those of 


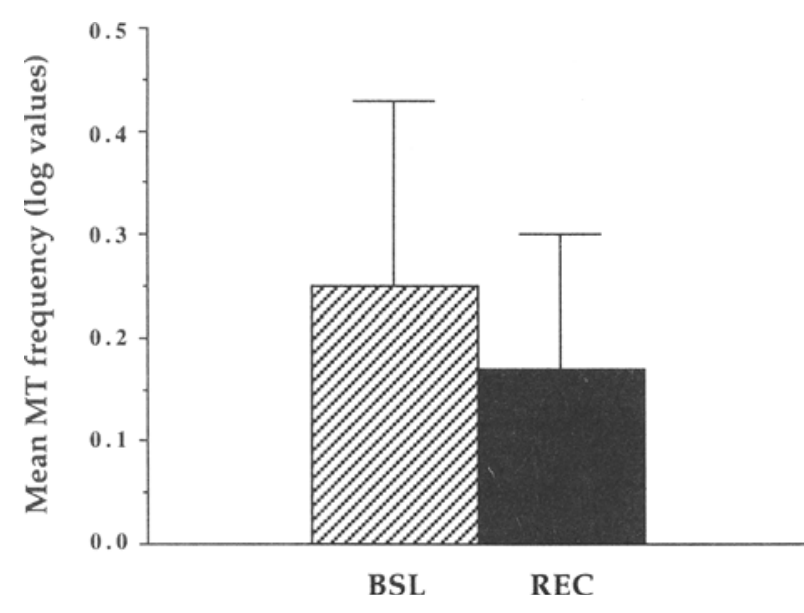

Figure 1. Mean muscle twitch (MT) frequency recorded during baseline (BSL) and recovery (REC) nights after total sleep deprivation.

20 other male subjects (mean age, 22.9 years) recorded in previous experiments in similar conditions (De Gennaro, Ferrara, Urbani, \& Bertini, 2000; Ferrara, De Gennaro, \& Bertini, 1999) were considered. Electrophysiological measures (EEG, EOG, EMG) of these additional subjects were recorded during baseline nights with the same procedure as that previously described; the only difference was that 10 of them (Ferrara et al., 1999) had recordings of only submental EMG. For this reason, intercorrelations were performed between REM frequency and MT frequency recorded on submental derivations.

\section{RESULTS}

\section{Effects of Total Sleep \\ Deprivation on Recovery Sleep}

Table 1 shows means, standard deviations, and analysis of variance (ANOVA) results for the sleep structure variables. As a result of sleep deprivation, the recovery nights were characterized by a decrease of Stage 1 , Stage 2 , and intrasleep waking; these decreases were paralleled by the increase of SWS and of the sleep efficiency index, without affecting REM percentages.

\section{Effect of Total Sleep \\ Deprivation on Muscle Twitch Activity}

A $4 \times 2 \times 4$, derivation ( 2 submental and 2 masseter EMGs) $\times$ night (baseline vs. recovery) $\times$ REM period (1st vs. 2nd vs. 3rd vs. 4th), repeated measure ANOVA was carried out on logarithmic values of MT frequency. Results showed a main effect for the derivation factor $[F(3,24)=4.30, p=.01]$, indicating a higher MT frequency recorded from submental derivations (submental $1=0.245$; submental $2=0.272$ ) than from masseter derivations (masseter $1=0.154$; masseter $2=0.160$ ), and a significant effect for the night factor $[F(1,8)=6.31, p=$ .04 ], indicating that MT frequency during the recovery night decreased, as compared with the baseline night (see Figure 1). No other main effect or interaction was significant.

\section{Time Course of Muscle Twitches and Rapid Eye Movements (REMs) Across REM Cycles}

To assess the time course of MTs during the night, data recorded on 29 subjects during undisturbed baseline nights were $\log$ transformed and submitted to a repeated measure ANOVA, with derivation (submental 1 vs. submental 2) $\times$ REM period (1st vs. 2nd vs. 3rd vs. 4th) as factors. The ANOVA showed a significant main effect for REM period $[F(3,84)=2.69, p=.05]$. Planned comparisons indicated that MT frequency during the first REM period was significantly lower than that during the third $(p=.01)$ and the fourth $(p=.04)$ REM periods (see Figure 2). No other main effect or interaction was significant.

In addition, log values of REM frequency (number of horizontal REMs/REM duration) scored on the same data pool were submitted to a one-way repeated measure ANOVA, with REM period (1st vs. 2nd vs. 3rd vs. 4th) as a factor. The ANOVA showed a significant difference among REM periods $[F(3,84)=5.52, p=.001]$; planned comparisons indicated that REM frequency during the first period was significantly lower than those during the second $(p=.008)$, third $(p=.0002)$, and fourth $(p=.01)$ periods (see Figure 3 ).

\section{Correlations Between Muscle \\ Twitches and Rapid Eye Movements}

Correlations between the frequencies of MTs and REMs were assessed within each REM period of the baseline nights ( 29 subjects). Table 2 shows that only in the first REM period was there a significant, but weak, correlation between MTs and REMs.

\section{DISCUSSION}

To the best of our knowledge, this is the first report of a decrease in MT frequency in the recovery night after total sleep deprivation, as compared with baseline nights.

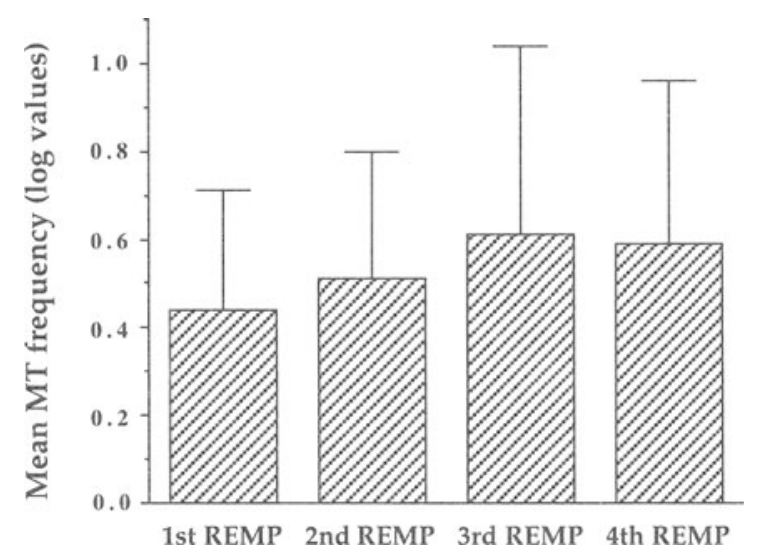

Figure 2. Time course of mean muscle twitch (MT) frequency across the first four rapid eye movement periods (REMPs) of nocturnal sleep. 


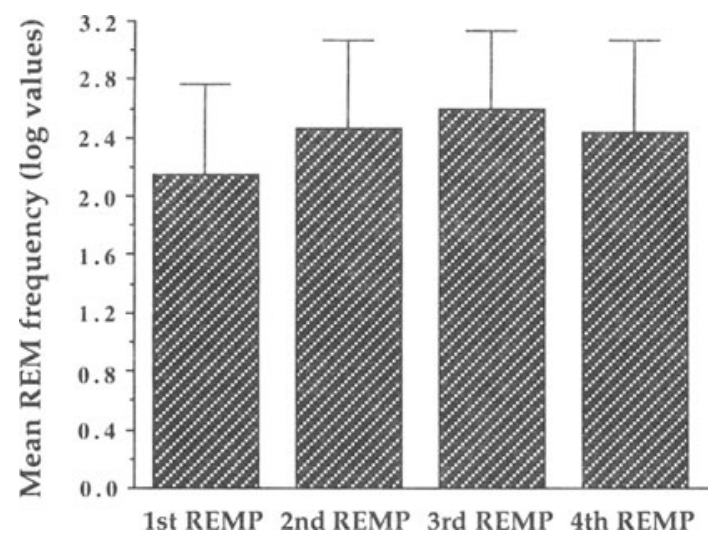

Figure 3. Time course of mean horizontal rapid eye movement (REM) frequency across the first four REM periods (REMPs) of nocturnal sleep.

Although of smaller size, this effect parallels the decrease of REM frequency during the same recovery nights (De Gennaro \& Ferrara, 2000), suggesting that an inverse relation also exists between MT activity and SWS increase after total sleep deprivation.

However, since it has been shown that, in the same recovery nights after total sleep deprivation, MEMA frequency - at variance with REM frequency-does not decrease, as compared with baseline (De Gennaro \& Ferrara, 2000), the hypothesis of a unique center of phasic motor activity during REM sleep (Morrison, Sanford, Ball, Mann, \& Ross, 1995) must be rejected.' Furthermore, the phasic discharge in various muscle groups during REM sleep cannot be considered, as was proposed by Geisler et al. (1987), as one "class" of phasic activity with common properties. Actually, these phasic phenomena are not only generated or triggered by different pathways (see, e.g., Hobson, Lydic, \& Baghdoyan, 1986), but are also modulated by different mechanisms, as is indicated by the following converging evidence: (1) the decrease in the smaller effect size of the MT after sleep deprivation, as compared with that of the REMs found in the present study; (2) the different time course of MTs and REMs across REM periods (REM frequency sharply increased during the second, third, and fourth REM periods, as compared with the first one, whereas MT frequency showed only a slight prevalence of the third and fourth periods, as compared with the first one); (3) the existence of a sig-

Table 2

Correlations Between Frequencies of Muscle Twitches and Rapid Eye Movements (REMs) Assessed Within Each REM Period of the Baseline Nights $(n=29)$

\begin{tabular}{ccl}
\hline REM Periods & Pearson's $r$ & $p$ \\
\hline 1st & .41 & .03 \\
2nd & .15 & .42 \\
3rd & .01 & .98 \\
4th & .11 & .58 \\
\hline
\end{tabular}

nificant correlation between REMs and MTs only in the first REM period; and (4) the absence of any variation in MEMA frequency across sleep cycles (De Gennaro \& Ferrara, 2000; Zarcone \& Benson, 1995).

As far as the intranight distribution pattern of MT activity is concerned, we found, at variance with the Geisler et al. (1987) and the Tafti et al. (1991) findings, that MT frequency during the first REM period was slightly but significantly lower than those during the third and fourth REM periods, whereas it did not differ from the second REM period. Actually, the discrepancy between our finding and those of Geisler et al. is limited to MT activity during the second REM period. This slight difference could be due to (1) the different age range of the two groups of subjects, our subjects being much younger than Geisler et al.'s subjects, and (2) the differences in calculating the frequency (or density) of phasic events. As regards the effects of age on MT density, Geisler et al. reported that the rate of MTs decreases significantly with age, although the specific effect of age on intranight distribution of MT is unknown. As regards the MT measurement procedure, we used an MT frequency measure, since we counted the single MTs and divided their number by the actual duration of REM sleep, whereas Geisler et al. used a measure of MT density by counting the number of 10 -sec periods containing MTs/minutes of REM sleep containing at least one MT. This latter procedure could have resulted in an underestimation of MT activity, whereas our procedure seems to allow a more accurate evaluation of these phasic events. In addition, a further source of variation between the two studies could come from the use by Geisler et al. of relative MT density values in describing the intranight distribution pattern of MTs. ${ }^{2}$

In conclusion, our results suggest that the robust inverse relationship between SWS and REM frequency can be extended to MT activity during REM sleep, although this latter relation is much weaker. Several findings converge in indicating that the hypothesis of a unique center of phasic motor activity during REM sleep has to be rejected MTs and REMs show some common features, since the inhibition of both phasic activities is most powerful in the beginning of the night, when they reveal a weak but significant relation. However, they do not seem to be modulated by a common mechanism, since the decrease of MT frequency after sleep deprivation is much smaller, as compared with REMs, the time course of MTs and REMs across REM periods is different, and the weak correlation between REMs and MTs is significant only in the first REM period.

\section{REFERENCES}

ASERINSKY, E. (1973). Relationship of rapid eye movements density to the prior accumulation of sleep and wakefulness. Psychophysiology, 10, $545-558$

De Gennaro, L., \& Ferrara, M. (2000). Sleep deprivation and phasic activity of REM sleep: A dissociation between rapid eye movements and middle-ear muscle activity. Sleep, 23, 81-85. 
De Gennaro, L., Ferrara, M., \& Bertini, M. (2000). The relationship between rapid eye movements frequency and slow-wave sleep rebound. Journal of Sleep Research, 9, 155-159.

De Gennaro, L., Ferrara, M., Urbani, L., \& Bertini, M. (2000). A complementary relationship between wake and REM sleep in the auditory system. Experimental Brain Research, 130, 105-112.

Feinberg, I., Baker, T., Leder, R., \& MarCh, J. D. (1988). Response of delta $(0-3 \mathrm{~Hz}$.) EEG and eye movements density to a night with 100 minutes of sleep. Sleep, 11, 473-487.

Feingerg, I., Floyd, T. C., \& MARCh, J. D. (1987). Effects of sleep loss on delta $(0-3 \mathrm{~Hz}$.) EEG and eye movements density: New observation and hypothesis. Electroencephalography \& Clinical Neurophysiology, 67, 217-221.

Ferrara, M., De Gennaro, L., \& Bertini, M. (1999). Selective slowwave sleep (SWS) deprivation and SWS rebound: Do we need a fixed SWS amount per night? Sleep Research Online, 2, 15-19. Available: www.SRO.org

Fridlund, A. J., \& CACIOPPO, J. T. (1986). Guidelines for human electromyographic research. Psychophysiology, 23, 567-589.

Geisler, P., Meier-Ewert, K., \& Matsubayshi, K. (1987). Rapid eye movements, muscle twitches and sawtooth waves in the sleep of narcoleptic patients and controls. Electroencephalography \& Clinical Neurophysiology, 67, 499-507.

Hobson, J. A., Lydic, R., \& BaGHDOYAN, H. (1986). Evolving concepts of sleep cycle generation: From brain centers to neuronal populations. Behavioral \& Brain Sciences, 9, 371-399.

Ktonas, P., Wu, F., \& Renault, H. (1992). Time-connectivity patterns in rapid eye movement occurrences during human REM sleep [Abstract]. Journal of Sleep Research, 1(Suppl. 1), 123.

Lucidi, F., Devoto, A., Violani, C., De Gennaro, L., Mastracci, P., \& BERTINI, M. (1996). Rapid eye movements density as a measure of sleep need: REM density decreases linearly with the reduction of prior sleep duration. Electroencephalography \& Clinical Neurophysiology, 99, 556-561.

Morrison, A. R., Sanford, L. D., Ball, W. A., Mann, G. L., \& Ross,
R. J. (1995). Stimulus-elicited behavior in rapid eye movement sleep without atonia. Behavioral Neuroscience, 109, 972-979.

Rechtschaffen, A., \& Kales, A. (1968). A manual of standardized terminology, techniques and scoring system for sleep stages of human subjects. Los Angeles: University of California, Brain Information Service, Brain Information Institute.

Reynolds, C. F., Kupfer, D. J., Hoch, C. C., Stack, J. A., Houch, P. R., \& BERMAN, S. R. (1986). Sleep deprivation in health elderly men and women: Effects on mood and on sleep during recovery. Sleep, 9, 492-501.

Tafti, M., Olivet, H., \& Billiard, M. (1991). Phasic events in narcolepsy and sleep apnea syndrome. In M. G. Terzano, P. L. Halasz, \& A. C Declerck (Eds.), Phasic events and dynamic organization (pp. 151166). New York: Raven.

Travis, F., Maloney, T., Means, M., March, J. D., \& Feinberg, I. (1991). Acute deprivation of the terminal four hours of sleep does not increase delta $(0-3 \mathrm{~Hz})$ electroencephalograms: A replication. Sleep, 14, 320-324.

Zarcone, V. P., \& BENSON, K. L. (1995). Middle ear muscle activity (MEMA) in schizophrenia using a noninvasive technique. Sleep, 18 , 266-271.

\section{NOTES}

1. According to this hypothesis, REMs, MEMA, MTs, and other phasic events of REM sleep could be considered as startle responses that may operate through a central motor command center (Morrison et al., 1995).

2. These values were calculated to compensate for the large interindividual differences in the density of MT and expressed the density of MT during each REM period in relation to the mean value for the whole night.

(Manuscript received December 21, 1999; revision accepted for publication March 9, 2000.) 